\title{
Upsurge of Enterovirus D68, the Netherlands, 2016
}

\section{Marjolein Knoester, Elisabeth H. Schölvinck, Randy Poelman, Sylvia Smit, Clementien L. Vermont, Hubert G.M. Niesters, Coretta C. Van Leer-Buter}

In June and July 2016, we identified 8 adults and 17 children with respiratory enterovirus D68 infections. Thirteen children required intensive care unit admission because of respiratory insufficiency, and 1 had concomitant acute flaccid myelitis. Phylogenetic analysis showed that all of 20 sequences obtained belong to the recently described clade B3.

$\mathrm{T}$ The largest enterovirus D68 (EV-D68) outbreak occurred in the United States during the summer and fall of 2014. Approximately 1,100 respiratory EV-D68 infections were recorded, mostly in children, many of whom required intensive care unit (ICU) admission. Most diseases were of respiratory nature, but concurrent with the upsurge of EV-D68, the Centers for Disease Control and Prevention (Atlanta, GA, USA) recorded 120 cases of acute flaccid myelitis (AFM) (1). A Europewide study identified circulation of EV-D68 during the same period. Although numbers were lower, ICU admissions and 3 cases of AFM were reported (2). Before 2014, only a few small outbreaks of EV-D68 had been described worldwide, all with respiratory infections.

Our clinical virology laboratory (University Medical Center Groningen, Groningen, the Netherlands) reported an increase of EV-D68 infections in 2010 and again in 2014 , in parallel with the US outbreak $(3,4)$. Very limited activity of EV-D68 was observed in 2015. Since June 2016, we have again identified a substantial increase in respiratory EV-D68 infections in our hospital, just 2 years after the previous upsurge. To raise awareness of this upsurge and the severity of EV-D68 infections, we report on 25 cases. In addition, we show the phylogenetic relationship between the 2016 EV-D68 strains and those that circulated in 2014.

\section{The Study}

The University Medical Center Groningen is a tertiary referral center in the northern part of the Netherlands. We perform routine diagnostic real-time PCR for all patients

Author affiliation: University Medical Center Groningen,

Groningen, the Netherlands

DOI: http://dx.doi.org/10.3201/eid2301.161313 evaluated for respiratory disease by laboratory developed tests for 17 targets, including adenovirus, bocavirus, coronavirus, enterovirus, metapneumovirus, influenza, parainfluenzavirus, rhinovirus, and respiratory syncytial virus (5). From all enterovirus detections with a cycle threshold $\left(\mathrm{C}_{\mathrm{t}}\right)$ value $<31$, we sequence part of the viral protein 1 gene on a weekly basis (6). To obtain rapid typing results in the current outbreak, a specific EV-D68 real-time PCR was also used (3), when enterovirus PCR was found positive.

We identified 3 EV-D68 infections in June and 22 additional cases in July. We found a stable seasonal variance for enterovirus and a sudden rise of enterovirus infections in July, mainly caused by EV-D68 (Figure 1).

The EV-D68-positive specimens were nasopharyngeal swabs $(\mathrm{n}=8)$, sputum $(\mathrm{n}=2)$, and nasopharyngeal brushes $(n=15)$. Two viral respiratory co-infections were detected: rhinovirus A15 in patient 12 (enterovirus $\mathrm{C}_{t} 17$, rhinovirus $C_{t} 21$ ) and parainfluenzavirus type 4 in patient 23 (enterovirus $C_{t} 14$, parainfluenza virus $4 C_{t} 31$ ). For 20 patients, we obtained sequence results identifying EV-D68; the remaining 5 could not be typed by sequencing but were positive in our specific EV-D68 PCR.

Of the 25 patients, 8 were adults and 17 were children (Table). In the adults, 6 of whom were transplant recipients, symptoms were mild and influenza-like. In children, however, we observed life-threatening respiratory distress, for which ICU admission was necessary for 13 (Table). Twenty-one patients had an underlying or concurrent condition, in the children mostly pulmonary.

One child (patient 16) had the clinical characteristics of AFM. This nearly 4-year-old boy had a history of headaches for 1 week and an influenza-like illness for 3 days. He had rapidly progressing asymmetric weakness of arms and legs, bulbar paralysis, asymmetric facial paralysis, and respiratory distress for which he needed ventilatory support. Cerebrospinal fluid (CSF) analysis showed no abnormalities. Axial fluid-attenuated inversion recovery magnetic resonance imaging (MRI) showed hyperintense nonenhancing gray matter lesions in brainstem and spinal cord. Electromyography findings supported injury on the level of the motor axon or the anterior horn of the spinal cord. No varicella zoster virus, herpes simplex virus, enterovirus, or parechovirus were detected in the CSF. In the nasopharyngeal swab, only EV-D68 was detected.

All patients in this report were treated in our tertiary referral hospital, but they originated from different regions in the north of the Netherlands. Spatial epidemiology data 


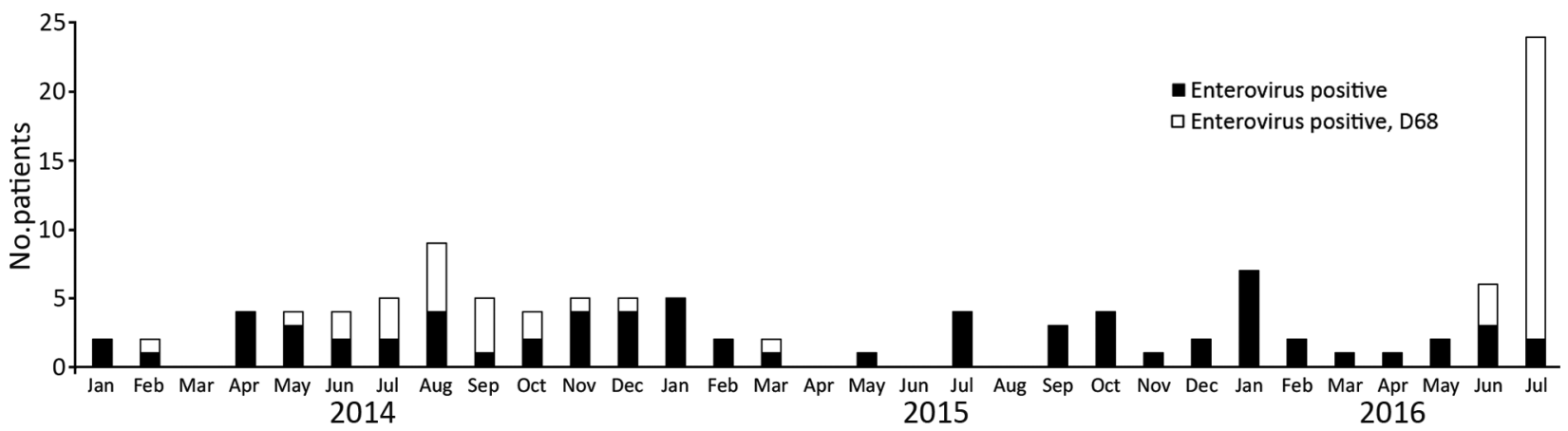

Figure 1. Respiratory enterovirus infections, University Medical Center Groningen, the Netherlands, 2014, 2015, and 2016. The total numbers of respiratory specimens tested for enterovirus in these years during April-July were 784 , 828, and 871 , respectively. Seventeen (2.2\%) of 784 were positive for enterovirus in 2014, $5(0.6 \%)$ of /828 in 2015, and $33(3.8 \%)$ of /871 in 2016 . Six (35\%) of 17 were typed as enterovirus D68 in 2014, versus 0 of 5 in 2015, and 25 (76\%) of 33 in 2016.

did not suggest an epidemiologic link or outbreak (data not shown). In 2 children, EV-D68 symptoms developed $>48$ hours after admission; the source of possible nosocomial infection was not identified.

We obtained 20 sequences of part of the EV-D68 viral protein gene and aligned them with the sequences from the 2014 epidemic. Clades were assigned as described previously (7) by the neighbor-joining method by using BioNumerics software 6.6 (Applied Maths/bioMérieux, Sint-Martens-Latem, Belgium). Sequence analysis (Figure 2) showed that the 2016 strains were closely related to sequences of the recently described subclade B3 (8),

\begin{tabular}{|c|c|c|c|c|c|}
\hline Patient & Date† & Age/sex & Concurrent condition(s) & Clinical characteristics & $\begin{array}{l}\text { ICU admission, } \\
\text { days in ICU }\end{array}$ \\
\hline 1 & Jun 11 & $1 \mathrm{y} / \mathrm{M}$ & None & Bronchial obstruction, respiratory failure & Yes/4 \\
\hline 2 & Jun 20 & $3 \mathrm{y} / \mathrm{M}$ & None & Status asthmaticus & Yes/3 \\
\hline 3 & Jun 30 & $61 \mathrm{y} / \mathrm{F}$ & Lung and liver transplantation & Cough, runny nose & No \\
\hline 4 & Jul 4 & $5 \mathrm{y} / \mathrm{M}$ & Asthma & Status asthmaticus & Yes/2 \\
\hline 5 & Jul 5 & $3 y / M$ & Asthma & Status asthmaticus & Yes/3 \\
\hline 6 & Jul 7 & $67 \mathrm{y} / \mathrm{F}$ & Autologous stem cell transplantation & Influenza-like syndrome, dyspnea & No \\
\hline 7 & Jul 7 & $2 \mathrm{y} / \mathrm{M}$ & $\begin{array}{l}\text { Pulmonary hypertension, } \\
\text { underdeveloped lung vessels }\end{array}$ & Respiratory failure & Yes/4 \\
\hline 8 & Jul 11 & $70 \mathrm{y} / \mathrm{F}$ & Allogenic stem cell transplantation & Influenza-like syndrome, dyspnea & No \\
\hline 9 & Jul 14 & $66 \mathrm{y} / \mathrm{F}$ & Allogenic stem cell transplantation & Cough, fever & No \\
\hline 10 & Jul 16 & $3 \mathrm{y} / \mathrm{M}$ & Asthma & Status asthmaticus & Yes/4 \\
\hline 11 & Jul 16 & $3 \mathrm{mo} / \mathrm{M}$ & $\begin{array}{l}\text { Bronchopulmonary dysplasia of } \\
\text { prematurity }\end{array}$ & Respiratory failure & Yesł \\
\hline 12 & Jul 16 & $1 \mathrm{y} / \mathrm{F}$ & Bronchial hyperreactivity, failure to thrive & Bronchial obstruction, respiratory failure & Yes/8 \\
\hline 13 & Jul 22 & $5 \mathrm{y} / \mathrm{F}$ & Asthma & Status asthmaticus & Yes/5 \\
\hline 14 & Jul 22 & $1 \mathrm{y} / \mathrm{M}$ & $\begin{array}{c}\text { Tracheal stoma for bilateral vocal cord } \\
\text { paralysis }\end{array}$ & Cough, wheezing & No \\
\hline 15 & Jul 23 & $3 \mathrm{y} / \mathrm{M}$ & None & Status asthmaticus & Yes/5 \\
\hline 16 & Jul 24 & $3 y / M$ & Atypical seizures 3 y prior & Acute flaccid myelitis & Yes/ongoing§ \\
\hline 17 & Jul 25 & $19 \mathrm{y} / \mathrm{F}$ & None & Influenza-like syndrome & No \\
\hline 18 & Jul 25 & 3 y/M & Bronchial hyperreactivity & Asthma exacerbation & No \\
\hline 19 & Jul 25 & $5 \mathrm{mo} / \mathrm{M}$ & $\begin{array}{l}22 q 11 \text { deletion, tetralogy of Fallot, } \\
\text { tracheal stoma }\end{array}$ & $\begin{array}{c}\text { Acute mechanical airway obstruction, } \\
\text { resuscitation }\end{array}$ & Noף \\
\hline 20 & Jul 28 & $50 \mathrm{y} / \mathrm{M}$ & Lung transplantation & Cough, runny nose & No \\
\hline 21 & Jul 28 & $3 \mathrm{y} / \mathrm{M}$ & Spinal muscular atrophy & Respiratory failure & Yes/5 \\
\hline 22 & Jul 29 & $9 \mathrm{mo} / \mathrm{M}$ & $\begin{array}{c}\text { Corrected esophagal atresia with related } \\
\text { tracheomalacy for which noninvasive } \\
\text { ventilation at home }\end{array}$ & Respiratory distress with bronchorrhea & No \\
\hline 23 & Jul 30 & $3 y / F$ & Bronchial hyperreactivity & Status asthmaticus & Yes/2 \\
\hline 24 & Jul 30 & $51 \mathrm{y} / \mathrm{F}$ & Autologous stem cell transplantation & Influenza-like syndrome & No \\
\hline 25 & Jul 31 & $23 \mathrm{y} / \mathrm{M}$ & Childhood asthma & Cough, dyspnea, nausea, vomiting & No \\
\hline
\end{tabular}

${ }^{*} \mathrm{EV}$, enterovirus; ICU, intensive care unit.

†Date of first EV-D68-positive specimen.

$\ddagger$ Patient 11 contracted EV-D68 in the neonatal ICU.

§Patient still in ICU as of December 6, 2016.

TPatient 19 acquired EV-D68 while admitted to the special care ward since birth. 


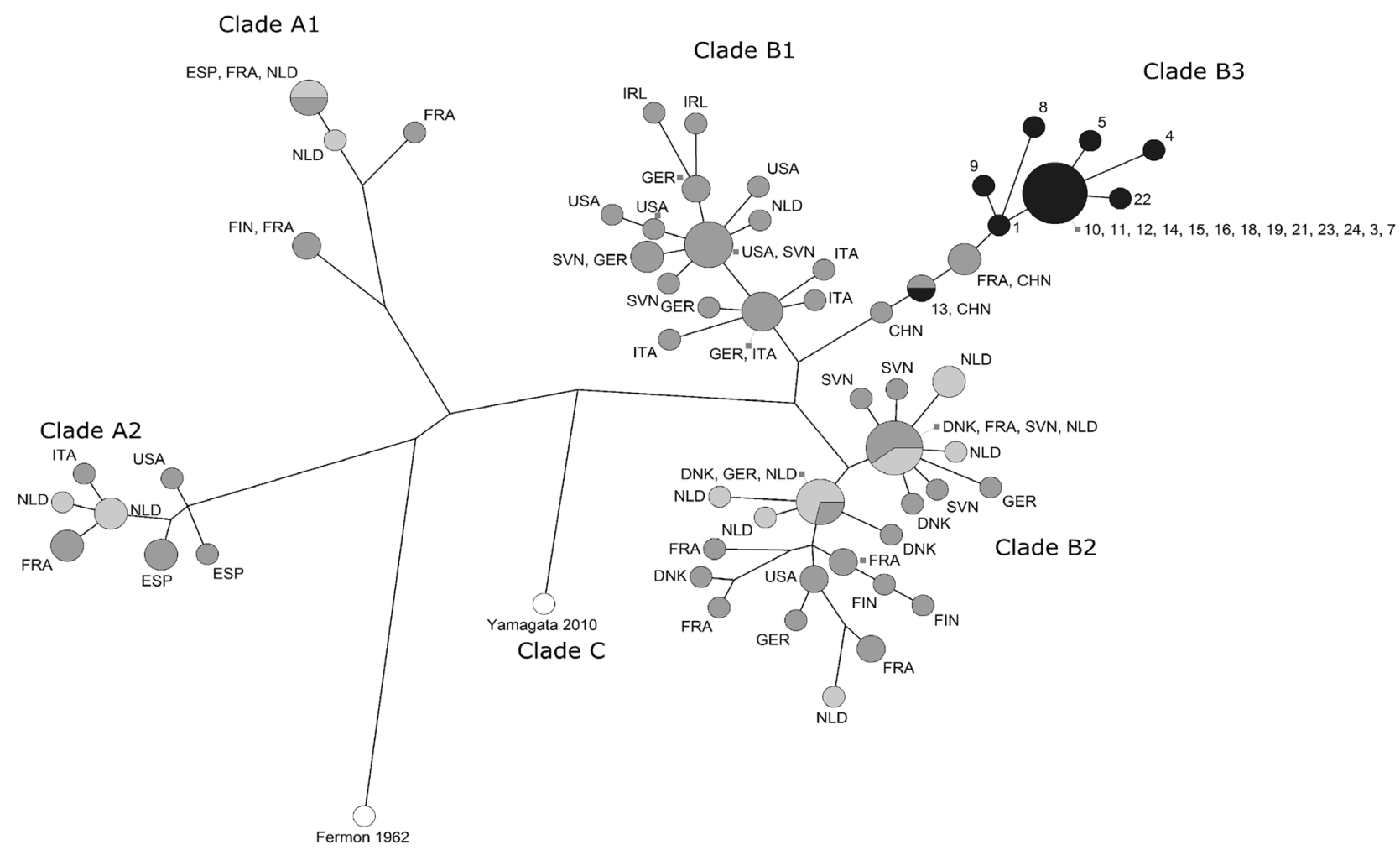

Figure 2. Maximum-parsimony tree of partial viral protein 1 sequences of enterovirus D68 (EV-D68). Included are the strains obtained in the laboratory of the University Medical Center Groningen (Groningen, the Netherlands) in 2014 (light gray, n = 23) and 2016 (black, $n=20$ ) and worldwide isolates from 2014 (dark gray, $n=73$ ). Recent strains cluster in the recently described clade B3, with a nucleotide divergence of $2.1 \%$ within clade B3, $5.5 \%$ to clade B1, and $7.3 \%$ to clade B2. Clades are according to Tokarz et al. and Gong et al $(7,8)$. We submitted the 20 sequences we obtained during 2016 to GenBank under accession nos.: KX685066-KX685084 and KX710328. CHN, China; DNK, Denmark; ESP, Spain; FIN, Finland; FRA, France; GER, Germany; IRL, Ireland; ITA, Italy; NLD, the Netherlands; SVN, Slovenia; USA, United States. White circles indicate reference strains $(n=2)$.

represented in Figure 2 by 4 sequences from China. The nucleotide divergence was $2.1 \%$ within $\mathrm{B} 3,5.5 \%$ between $\mathrm{B} 1$ and $\mathrm{B} 3$, and $7.3 \%$ between $\mathrm{B} 2$ and $\mathrm{B} 3$. We submitted our 20 sequences from 2016 to GenBank (accession nos. KX685066-KX685084 and KX710328).

\section{Conclusions}

After the upsurge of EV-D68 in our region during 2009 and 2010, we set up routine genotypic characterization for several viral pathogens, including enterovirus and rhinovirus, which is also offered to regional referring hospitals and healthcare institutions. Sequencing results are available within 1 week, which provides clinically relevant and epidemiologic information. This so-called REGIOTYPE strategy contributed to timely detection of EV-D68 in our hospital in 2014, as well as in 2016. We recognized a sudden reappearance of EV-D68 with a sharp increase of cases that could not be explained by a change in surveillance strategy.

In line with previous outbreaks, mostly children were affected, and those with underlying pulmonary conditions seemed at higher risk for ICU admission (9). The number of children who needed ICU admission was higher in 2016 than in our 2010 and 2014 reports $(3,4)$. Although dynamics of viral disease and shedding are not known for EVD68, we assume a role for EV-D68 in the symptoms of patients 12 and 23, as, in line with rhinovirus infections, higher viral load might indicate symptomatic disease (10).

Evidence that EV-D68 might cause AFM is increasing after recent epidemiologic investigations $(1,11,12)$. In patient 16, atypical Guillain-Barré syndrome initially was diagnosed; however, this diagnosis was later discarded because the electromyography results indicated motoric axon or anterior horn cell disease, and the clinical picture and MRI results were in favor of AFM (13). MRI findings were subtle, and radiologic diagnosis was made only after further review and discussion of the case with the neurologists. The absence of EV-D68 in CSF is consistent with previous reports $(1,12)$.

Sequencing results showed that the strains in our study cluster in the recently described clade B3 (8). During the 2014 outbreak, most EV-D68 sequences belonged to clades $\mathrm{B} 1$ and $\mathrm{B} 2$, although $\mathrm{A} 1$ and $\mathrm{A} 2$ were also represented 
$(2,14)$. Larger epidemiologic and genotyping studies are needed to evaluate whether the distinction within clade B is tenable and whether our clinical findings are typical for subclade B3.

This upsurge could indicate an active EV-D68 season, as highlighted by the epidemiologic curve, with a potential increase in AFM cases. Clinicians should be alert for EVD68, its clinical implications, and the need for appropriate diagnostics, particularly in children who are admitted with respiratory failure to the ICU or with possible symptoms of AFM.

\section{Acknowledgments}

We thank Oebo Brouwer for his neurology expertise.

Dr. Knoester is an attending physician in clinical microbiology at the Department of Medical Microbiology, University Medical Center Groningen. Her research focuses on clinical virology and epidemiology.

\section{References}

1. Sejvar JJ, Lopez AS, Cortese MM, Leshem E, Pastula DM, Miller L, et al. Acute flaccid myelitis in the United States, AugustDecember 2014: results of nation-wide surveillance. Clin Infect Dis. 2016;63:737-45. http://dx.doi.org/10.1093/cid/ciw372

2. Poelman R, Schüffenecker I, Van Leer-Buter C, Josset L, Niesters HG, Lina B; ESCV-ECDC EV-D68 study group. European surveillance for enterovirus D68 during the emerging North-American outbreak in 2014. J Clin Virol. 2015;71:1-9. http://dx.doi.org/10.1016/j.jcv.2015.07.296

3. Poelman R, Schölvinck EH, Borger R, Niesters HG, van Leer-Buter $\mathrm{C}$. The emergence of enterovirus D68 in a Dutch University Medical Center and the necessity for routinely screening for respiratory viruses. J Clin Virol. 2015;62:1-5. http://dx.doi.org/10.1016/j.jcv.2014.11.011

4. Rahamat-Langendoen J, Riezebos-Brilman A, Borger R, van der Heide R, Brandenburg A, Schölvinck E, et al. Upsurge of human enterovirus 68 infections in patients with severe respiratory tract infections. J Clin Virol. 2011;52:103-6. http://dx.doi.org/10.1016/j.jcv.2011.06.019

5. Bosis S, Esposito S, Niesters HGM, Zuccotti GV, Marseglia G, Lanari M, et al. Role of respiratory pathogens in infants hospitalized for a first episode of wheezing and their impact on recurrences. Clin Microbiol Infect. 2008;14:677-84. http://dx.doi.org/10.1111/j.1469-0691.2008.02016.x

6. Nix WA, Oberste MS, Pallansch MA. Sensitive, seminested PCR amplification of VP1 sequences for direct identification of all enterovirus serotypes from original clinical specimens. J Clin Microbiol. 2006;44:2698-704. http://dx.doi.org/10.1128/ JCM.00542-06

7. Tokarz R, Firth C, Madhi SA, Howie SR, Wu W, Sall AA, et al. Worldwide emergence of multiple clades of enterovirus 68. J Gen Virol. 2012;93:1952-8. http://dx.doi.org/10.1099/vir.0.043935-0

8. Gong YN, Yang SL, Shih SR, Huang YC, Chang PY, Huang CG, et al. Molecular evolution and the global reemergence of enterovirus D68 by genome-wide analysis. Medicine (Baltimore). 2016;95:e4416. http://dx.doi.org/10.1097/MD.0000000000004416

9. Messacar K, Abzug MJ, Dominguez SR. 2014 outbreak of enterovirus D68 in North America. J Med Virol. 2016;88:739-45. http://dx.doi.org/10.1002/jmv.24410

10. Jansen RR, Wieringa J, Koekkoek SM, Visser CE, Pajkrt D, Molenkamp R, et al. Frequent detection of respiratory viruses without symptoms: toward defining clinically relevant cutoff values. J Clin Microbiol. 2011;49:2631-6. http://dx.doi.org/ 10.1128/JCM.02094-10

11. Aliabadi N, Messacar K, Pastula DM, Robinson CC, Leshem E, Sejvar JJ, et al. Enterovirus D68 infection in children with acute flaccid myelitis, Colorado, USA, 2014. Emerg Infect Dis. 2016;22:1387-94. http://dx.doi.org/10.3201/eid2208.151949

12. Messacar K, Schreiner TL, Van Haren K, Yang M, Glaser CA, Tyler KL, et al. Acute flaccid myelitis: A clinical review of US cases 2012-2015. Ann Neurol. 2016;80:326-38. http://dx.doi.org/10.1002/ana.24730

13. Miller L. Council of State and Territorial Epidemiologists. Standardized case definition for acute flaccid myelitis. 2015 [cited 2016 Aug 20]. http://c.ymcdn.com/sites/www.cste.org/resource/ resmgr/2015PS/2015PSFinal/15-ID-01.pdf

14. Schuffenecker I, Mirand A, Josset L, Henquell C, Hecquet D, Pilorgé L, et al. Epidemiological and clinical characteristics of patients infected with enterovirus D68, France, July to December 2014. Euro Surveill. 2016;21:21. http://dx.doi.org/10.2807/15607917.ES.2016.21.19.30226

Address for correspondence: Marjolein Knoester, Department of Medical Microbiology, Division of Clinical Virology, University Medical Center Groningen, Hanzeplein 1, Groningen 9713 GZ, the Netherlands email: m.knoester@umcg.nl 